\title{
Structural relaxation and low-energy properties of twisted bilayer graphene
}

\author{
Giovanni Cantele $\odot,{ }^{1, *}$ Dario Alfè, ${ }^{2,3}$ Felice Conte, ${ }^{3,1}$ Vittorio Cataudella, ${ }^{3,1}$ Domenico Ninno, ${ }^{3,1}$ and Procolo Lucignano ${ }^{3, \dagger}$ \\ ${ }^{1}$ CNR-SPIN, c/o Complesso di Monte S. Angelo, via Cinthia, 80126 Napoli, Italy \\ ${ }^{2}$ Department of Earth Sciences and London Centre for Nanotechnology, University College London, \\ Gower Street, London WC1E 6BT, United Kingdom \\ ${ }^{3}$ Università degli Studi di Napoli “Federico II,” Dipartimento di Fisica “Ettore Pancini," \\ Complesso di Monte S. Angelo, via Cinthia, 80126 Napoli, Italy
}

(Received 28 April 2020; revised 29 July 2020; accepted 31 August 2020; published 23 October 2020)

\begin{abstract}
The structural and electronic properties of twisted bilayer graphene are investigated from first-principles and tight-binding approach as a function of the twist angle (ranging from the first "magic" angle $\theta=1.08^{\circ}$ to $\theta=13.17^{\circ}$, with the former corresponding to the largest unit cell, comprising 11,164 carbon atoms). By properly taking into account the long-range van der Waals (vdW) interaction, we provide the patterns for the atomic displacements (with respect to the ideal twisted bilayer). The out-of-plane relaxation shows an oscillating ("buckling") behavior, very evident for the smallest angles, with the atoms around the AA stacking regions interested by the largest displacements. The out-of-plane displacements are accompanied by a significant in-plane relaxation, showing a vortexlike pattern, where the vorticity (intended as curl of the displacement field) is reverted when moving from the top to the bottom plane and vice versa. Overall, the atomic relaxation results in the shrinking of the AA stacking regions in favor of the more energetically favorable $\mathrm{AB} / \mathrm{BA}$ stacking domains. The measured flat bands emerging at the first magic angle can be accurately described only if the atomic relaxations are taken into account. Quite importantly, the experimental gaps separating the flat-band manifold from the higher and lower energy bands are intimately related to out-of-plane relaxations. The stability of the relaxed bilayer at the first magic angle is estimated to be of the order of $0.5-0.9 \mathrm{meV}$ per atom (or 7-10 K). Our calculations shed light on the importance of an accurate description of the vdW interaction and of the resulting atomic relaxation to envisage the electronic structure of this really peculiar kind of vdW bilayers.
\end{abstract}

DOI: 10.1103/PhysRevResearch.2.043127

\section{INTRODUCTION}

After the first experimental findings [1,2], twisted bilayer graphene (TBG) has been the subject of intense investigation from both an experimental and theoretical point of view. When the rotation angle between the two graphene layers is close to the first "magic angle," $\theta \sim 1.08^{\circ}$, transport experiments show different superconducting domes as well as correlated insulating phases [3-8].

Most of the unconventional transport properties of TBG originate from the almost flat bands (FBs) at the Fermi energy, originally predicted in Ref. [9], whose bandwidth, of the order of $\sim 10 \mathrm{meV}$, has been confirmed also from tunnel spectroscopy experiments [8,10-12]. The FBs' manifold, which can host up to four electrons above the Fermi energy and four holes below it, is separated by an energy gap of $\sim 50 \mathrm{meV}$ from both higher and lower energy bands and has been clearly observed in recent nano-ARPES measurements [13]. When an external gate tunes the system chemical potential within

\footnotetext{
*giovanni.cantele@spin.cnr.it

†procolo.lucignano@unina.it
}

Published by the American Physical Society under the terms of the Creative Commons Attribution 4.0 International license. Further distribution of this work must maintain attribution to the author(s) and the published article's title, journal citation, and DOI. these gaps, a clear band insulating phase appears. A second, unexpected, insulating phase shows up at half-filling of the FB manifold, both on the electron and on the hole side $( \pm 2$ electrons with respect to charge neutrality). The correlated insulating phase is attributed to enhanced electron-electron interaction within the FBs [14,15], although some authors are highlighting the relevance of the electron-phonon interaction [16-19]. After electrostatic doping, achieved by gating the structure, unconventional superconductivity with a critical temperature ranging from 1.7 to $3 \mathrm{~K}$ appears in a strong pairing regime, with a phase diagram very similar to that of the underdoped cuprates, whose origin is still to be understood $[17,20]$.

Similar physics is being addressed also in twisted bilayers made out of transition-metal dichalcogenides [21], germanium selenide [19], and other two-dimensional materials [22-24]. That reveals how the twist angle can be used as a further degree of freedom [25] for combining two-dimensional (2D) materials to implement desired properties [26-30]. The twisted lattice geometry can be responsible for complex electron dynamics [31] and give rise to topological properties of TBG [32-34], unlike conventional topological materials [35], where topological properties are mostly due to spin-orbit interactions [36,37] and Brillouinzone topology.

In this paper we apply large-scale density functional calculations, accurately taking into account the interlayer van der 
Waals interaction, to better elucidate the origin of the FBs in the single-particle band structure and show the fundamental role played by the atomic relaxation. Relaxation mechanisms have been recently addressed using semiclassical [38-41], multiscale [42-44], or continuum relaxation [45] techniques. Uchida et al. [46] carried out large-scale density functional calculations within a real-space scheme in the local density approximation (with the van der Waals interaction taken into account only for larger angles). On the other hand, a fundamental advance using the effective continuum theory that will be addressed in the next sections was obtained by Nam and Koshino [40,47], who introduced lattice relaxation effects within a low-energy approximation. Quite recently, Carr et al. [48] incorporated the effects of the lattice relaxation within an $a b$ initio $k \cdot p$ perturbation continuum model. By contrast, in this manuscript we resort to a density functional theory (DFT) approach already presented in Ref. [49] and find new relaxation patterns, both at the magic angle and in other low-angle twisted structures. The properties of the FB manifold, at the first magic angle, are connected with the atomic displacements originating from the interlayer van der Waals interaction. We show that the energy gain induced by the relaxation becomes of the order of $10 \mathrm{~K}$, much larger than the typical temperature at which unconventional superconductivity or the correlated insulating phase are observed in TBG $(T<1 \div 2 \mathrm{~K})$. The smaller the twist angle, the more pronounced the atomic displacements with respect to the flat bilayer. In particular, we single out an oscillating displacement pattern of the out-of-plane displacements at smaller angles, which is smoothed at the larger angles, and a vortexlike in-plane displacement pattern where the atoms "rotate" in opposite directions in the two planes.

Tight-binding calculations, both at the relaxed and unrelaxed positions, are also carried out to provide a further and less expensive tool to reproduce, especially at the first magic angle, the electronic structure. We also give the effective parameters that best approximate the $a b$ initio band structure within the low-energy continuum theory [9], generalized in the presence of atomic relaxation [50]. Interestingly enough, they are largely independent of the twist angle, which makes the continuum model an excellent tool to describe the lowenergy physics at small twist angles.

The paper is organized as follows: In Sec. II we outline the technical details of the calculations. In Sec. III we extensively discuss the results on the geometrical relaxation and displacement patterns. In Sec. IV the tight-binding approach and the continuum model outcomes are compared with the $a b$ initio band structure. Finally, in Sec. V we summarize our findings and draw our final conclusions.

\section{METHODS}

DFT calculations have been carried out using the Vienna $\mathrm{Ab}$ initio Simulation Package (VASP) [51]. The vdW-DF2 exchange-correlation functional [52] has been adopted to properly take into account the long-range interactions taking place between atoms belonging to different graphene layers. A projector augmented wave (PAW) pseudopotential $[53,54]$ has been employed for carbon with the $2 p$ orbitals in valence and the $1 s$ orbitals frozen in the core. The single-particle Bloch waves were expanded with a plane-wave basis set using a cutoff energy of $400 \mathrm{eV}$.

TBG systems corresponding to four twist angles have been considered: $\theta=1.08^{\circ}, \theta=1.61^{\circ}, \theta=2.65^{\circ}$, and $\theta=$ $3.698^{\circ}$. The rotation is carried out starting from two perfectly AA stacked graphene layers and rotating around an axis orthogonal to the layers and passing through two $\mathrm{C}$ atoms, one on top of the other, belonging to the two planes (that therefore preserve their initial AA stacking). The respective structures can also be classified, according to the notation commonly used in the literature [55], using the pair of indexes $(n, m)$ : $(31,30),(21,20),(13,12)$, and $(9,8)$, respectively. The corresponding supercells comprise 11164, 5044, 1876, 868 atoms with lattice parameter $a=129.75,87.21,53.19$, and 36.18 $\AA$, respectively. For better elucidating the behavior of some properties at large twist angles, we have also considered, in some calculations, supercells with twist angles up to $13.17^{\circ}$.

Sampling of the Brillouin zone (BZ) for the self-consistent (SCF) calculations was restricted at the $\Gamma$ point for all four systems; no significant changes were observed after increasing the size of the sampling of the BZ for the smaller supercells. Single-particle energies at other points in the BZ were obtained by non-SCF calculations.

For the smallest angle, because of the size of the simulation cell we could only compute one $k$ point at a time, and the reported single-particle energies were therefore referred to the Fermi energy computed in the SCF calculation. The size of the supercell in the direction orthogonal to the layers ( $z$ axis) was initially fixed at $10 \AA$, corresponding to about $6.5 \AA$ vacuum space, introduced to prevent periodic replicas of the TBG supercell from interacting with each other. Full relaxation of the atomic positions was carried out until the residual forces were smaller than $0.002 \mathrm{eV} / \AA$. Additional calculations were repeated using supercells with $z$ axis of 12 and $14 \AA$. A small residual (maximum) relaxation of less than $0.002 \AA$ was observed as the $z$ axis was increased to $12 \AA$, but no further relaxation was detectable with the largest $14-\AA$ vacuum space. All symmetries were turned off. Further details on the calculations can be found in our previous paper [49].

Tight-binding calculations of the TBG electronic structure at different twist angles and geometries were also carried out using the Slater-Koster tight-binding parametrization for $p_{z}$ carbon atoms:

$$
\begin{aligned}
& t(R)=-V_{p p \pi}\left[1-\left(\frac{\mathbf{R} \cdot \hat{\mathbf{z}}}{R}\right)^{2}\right]-V_{p p \sigma}\left(\frac{\mathbf{R} \cdot \hat{\mathbf{z}}}{R}\right)^{2}, \\
& V_{p p \pi}=V_{p p \pi}^{0} e^{\left(R-a_{0}\right) / r_{0}}, \quad V_{p p \sigma}=V_{p p \sigma}^{0} e^{\left(R-d_{0}\right) / r_{0}} .
\end{aligned}
$$

Here $r_{0}=0.184 a$ is the decay length of the transfer integral, $a_{0}=a / \sqrt{3}$ is the first-neighbor distance in graphene, and $d_{0}=0.335 \mathrm{~nm}$ is the intralayer distance, chosen in agreement with that of graphite. $V_{p p \pi}^{0}=-2.7 \mathrm{eV}$ and $V_{p p \sigma}^{0}=0.48 \mathrm{eV}$ are the in-plane and out-of plane nearest-neighbor hopping energy, as from Ref. [56].

We also adopt a continuum model generalizing the model proposed in Refs. [9,50,56,57], providing an effective lowenergy band structure. Within this approach, the two planes are coupled via two overlap coefficients $u, u^{\prime}$ that can be expressed as integrals involving the tight-binding hopping term $t(R)$ of Eq. (1). The special case $u=u^{\prime}$ corresponds to the 


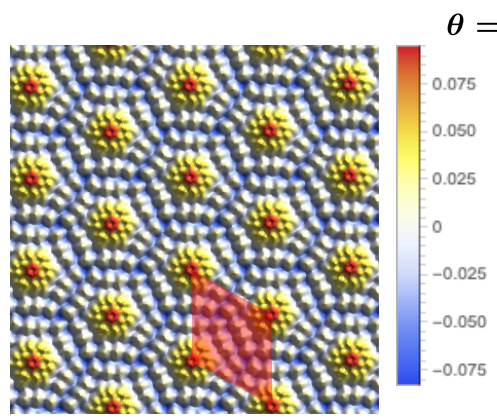

(a)

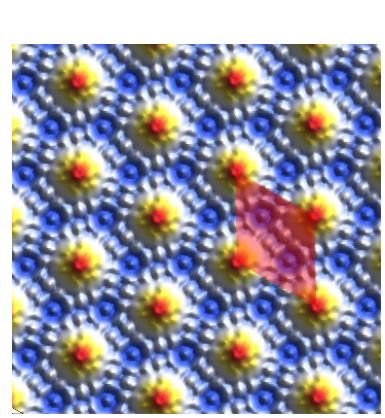

(c)

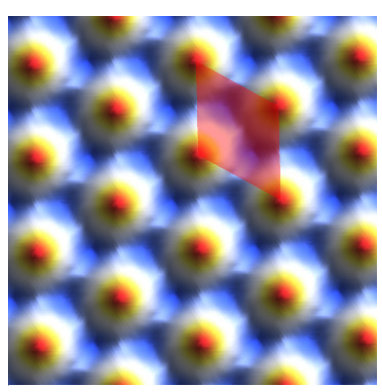

(e)

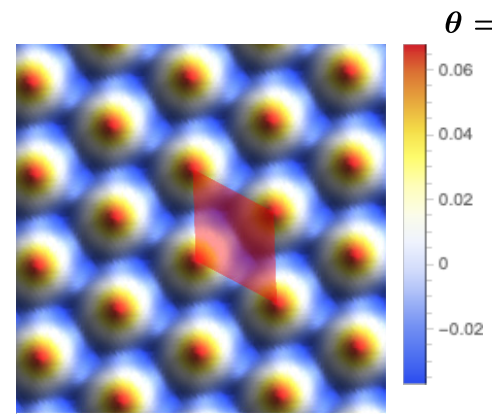

(g)

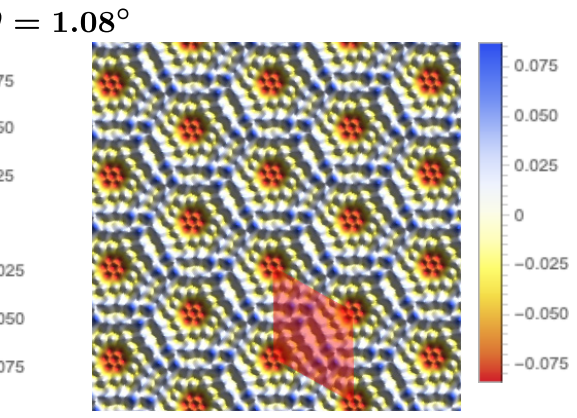

(b)

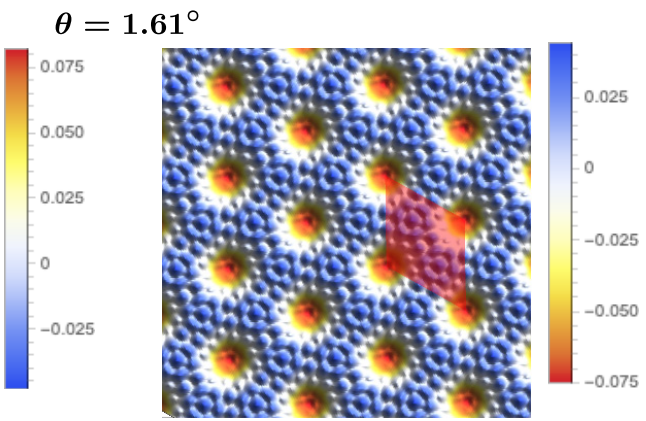

(d)

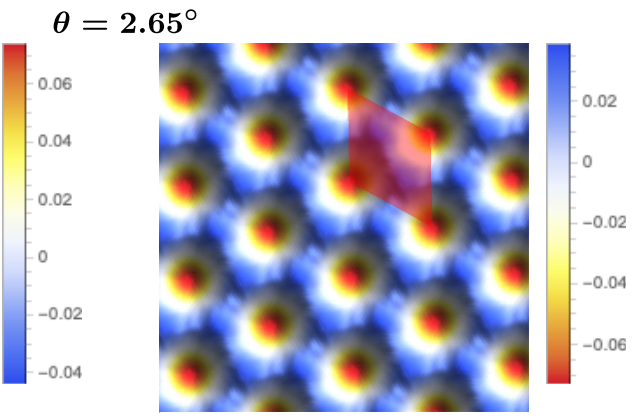

(f)

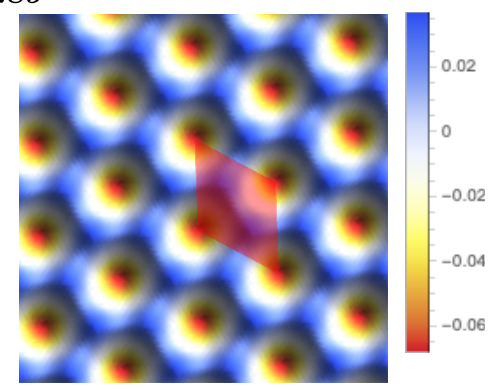

(h)

FIG. 1. The color/relief map of the out-of-plane displacements in TGB at different twist angles: (a, b) $1.08^{\circ},(\mathrm{c}, \mathrm{d}) 1.61^{\circ},(\mathrm{e}, \mathrm{f}) 2.65^{\circ}$, $(\mathrm{g}, \mathrm{h}) 3.89^{\circ}$. (a), (c), (e), and (g) refer to the top plane, whereas (b), (d), (f), and (h) to the bottom one. The color bar reports, in each plane, the measure of the $z$ coordinate referred to its mean value in that plane (in $\AA$ units). In each map the unit cell is highlighted in red.

unrelaxed graphene bilayer. In order to give a minimal model capable of describing (at least) the low-energy properties of the $a b$ initio band structure, we do not calculate $u, u^{\prime}$ but use them as fitting parameters. In the following we will show that the fitted parameters are relatively close to (but quantitatively different from) those obtained performing the hopping integrals [49]. Remarkably, we will show that $u, u^{\prime}$ can be chosen almost independently of the twist angle. 

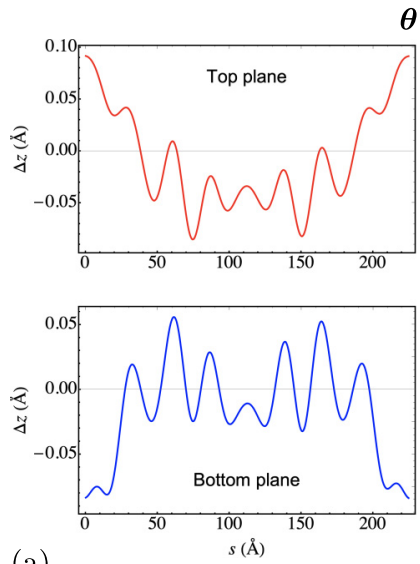

(a) $=1.08^{\circ}$
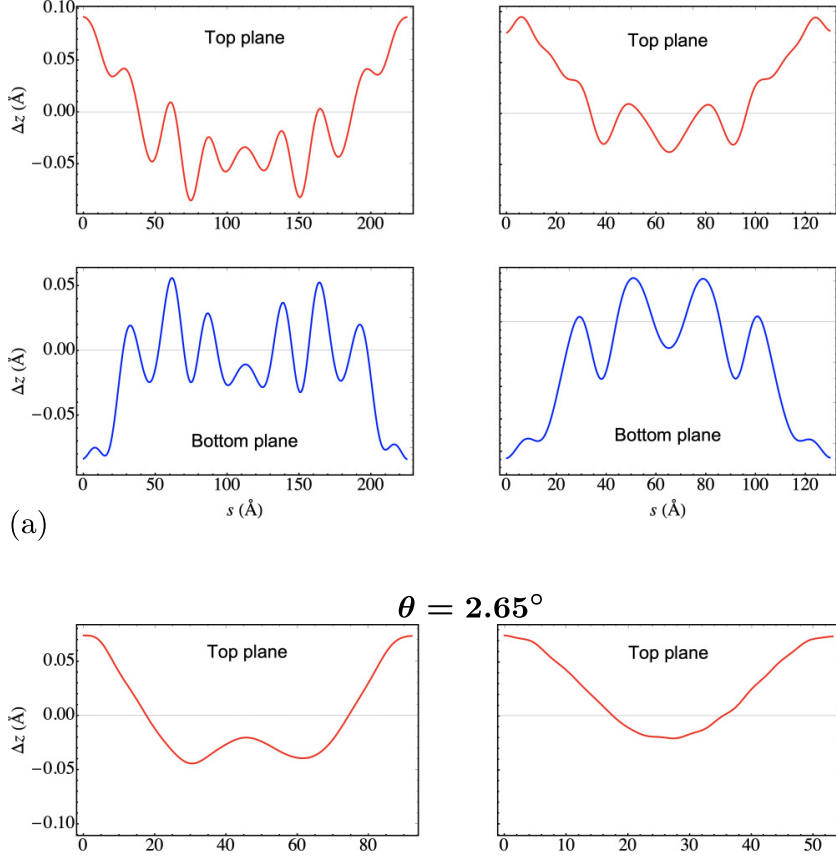

$\theta=2.65^{\circ}$
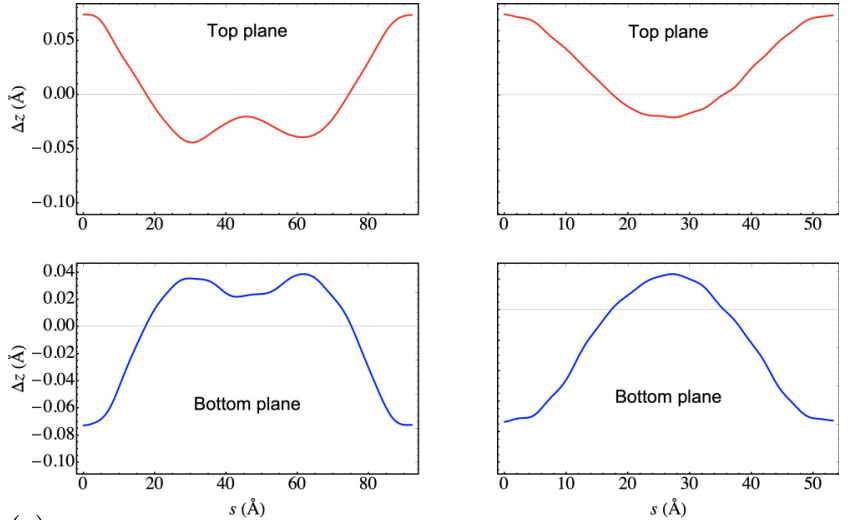

(c)

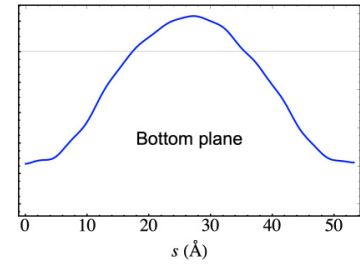

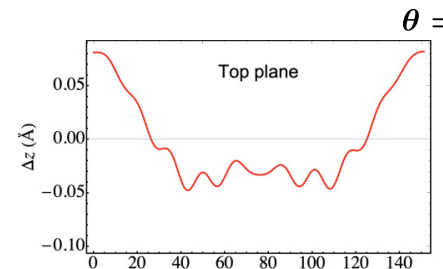

$\theta=1.61^{\circ}$
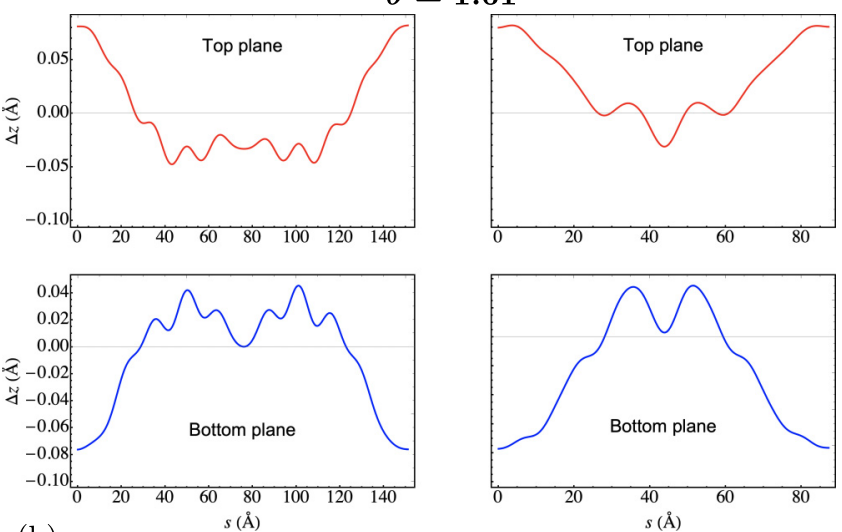

(b)

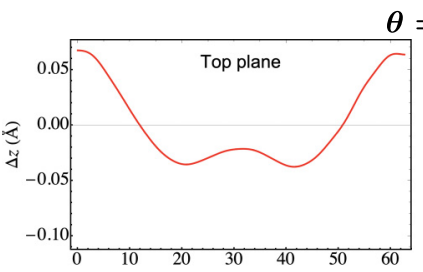

$\theta=3.89^{\circ}$
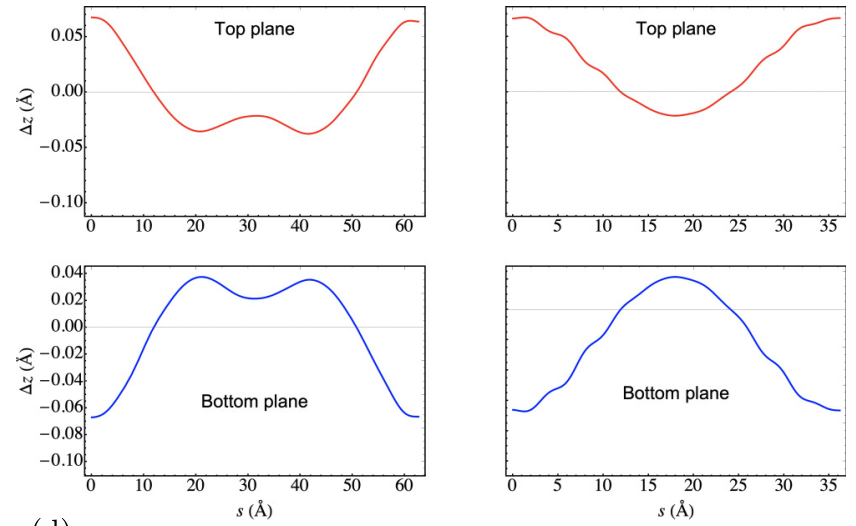

(d)

FIG. 2. The out-of-plane displacements of each graphene plane along the unit-cell long and short diagonal for the four twist angles: $\theta=1.08^{\circ}(\mathrm{a}), \theta=1.61^{\circ}(\mathrm{b}), \theta=2.65^{\circ}(\mathrm{c}), \theta=3.898^{\circ}(\mathrm{d})$. The displacements are measured as the difference $\Delta z=z-z_{\text {avg }}$ of the $z$ coordinate of each atom in a given plane and the average $z$ coordinate $z_{\text {avg }}$ in that plane $(\Delta z=0$ for all atoms in the initial, twisted but unrelaxed configuration). In each box the higher (lower) panels correspond to the top (bottom) plane, whereas the left (right) panels correspond to the supercell long (short) diagonal (notice the different scale on the horizontal axis). $s$ represents the coordinate along the two diagonals, with $s=0$ corresponding in both cases to the unit-cell origin (or a lattice equivalent site), where the AA stacking is preserved. To draw this plot, the atoms whose projections in the $x-y$ plane lie onto or are closest to the unit-cell diagonals were considered. Notice that the same direction was considered in the bottom and top plane; hence the $z$ displacements in the two planes should not be expected to be mirror symmetric one with respect to the other.

\section{GEOMETRIC RELAXATION}

Through a proper inclusion, within the ab initio approach, of the long-range interlayer vdW interaction, we provide a detailed and accurate description of the atomic relaxations arising from the interlayer interaction. We start by discussing the out-of-plane atomic displacements. We give two complementary representations in Figs. 1 and 2. The former shows a color/relief map of the atomic displacements with respect to the ideal, unrelaxed structure. To properly understand the results, we should recall that the TBG is built up starting from an ideal AA stacked bilayer and then rotating around an axis orthogonal to the graphene planes and passing through to two atoms, each belonging to a different layer. After twisting, these latter atoms preserve the original AA stacking, which is kept also after relaxation. However, due to the steric repulsion of their $p_{z}$ orbitals, they move far apart from each other. This
TABLE I. Energy gain induced by the structural relaxation as depicted in Figs. 1 and 3 for TBG at the four considered twist angles. $N_{\text {atoms }}$ is the number of atoms in each system, $\Delta E_{\text {relax }}$ the difference between the energy of TBG at the optimized geometry and that of the same bilayer in the initial configuration (twisted but unrelaxed graphene bilayer). The optimized distance $d$ between the two graphene planes, computed as the difference between the averages of the $z$ coordinates in the two planes, is also reported.

\begin{tabular}{lcccc}
\hline \hline & $(9,8)$ & $(13,12)$ & $(21,20)$ & $(31,30)$ \\
$N_{\text {atoms }}$ & 868 & 1876 & 5044 & 11164 \\
$\Delta E_{\text {relax }}(\mathrm{meV})$ & -0.513 & -1.157 & -3.657 & -9.797 \\
$\Delta E_{\text {relax }} / N_{\text {atoms }}(\mathrm{meV})$ & -0.590 & -0.617 & -0.725 & -0.878 \\
$\Delta E_{\text {relax }} / N_{\text {atoms }}(\mathrm{K})$ & 6.9 & 7.2 & 8.4 & 10.2 \\
$d(\AA)$ & 3.438 & 3.434 & 3.425 & 3.408 \\
\hline \hline
\end{tabular}


(a)

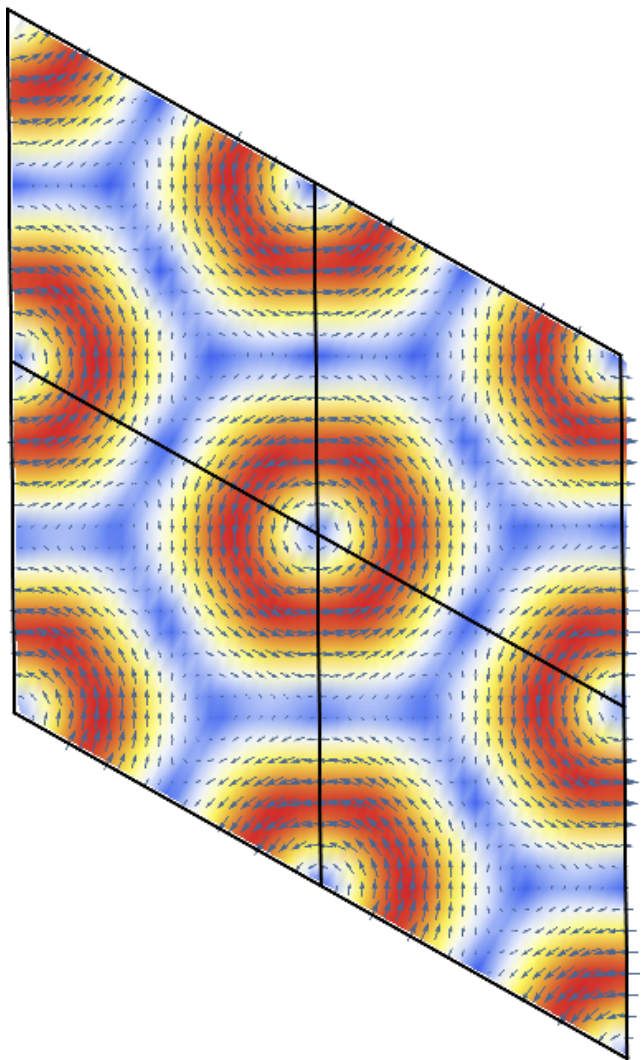

(c)

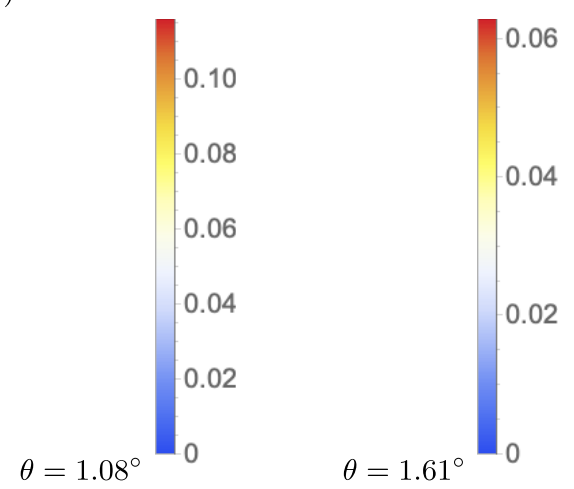

(b)
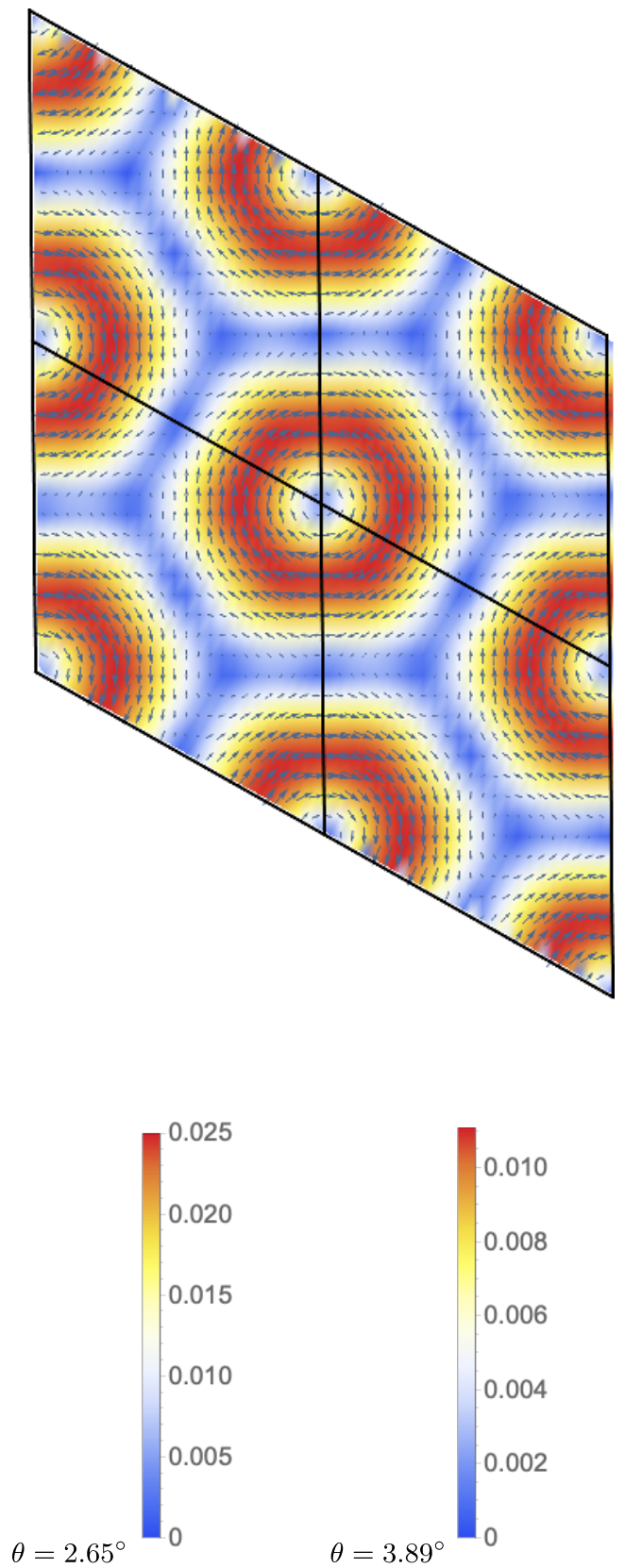

FIG. 3. The color map and vector field of the in-plane displacements in TGB for the top (a) and bottom (b) plane. Each vector points in the displacement direction, and its magnitude is proportional to the displacement norm. The latter is also highlighted by the color. Since the patterns look quite similar at different twist angles, only the result for $1.08^{\circ}$ is shown. (c) The color bars report the measure of the displacement in the $x-y$ plane with respect to the unrelaxed structure (in $\AA$ units).

corresponds to the hills (valleys) in the top (bottom) plane, clearly visible in the (a), (c), (e), (g) [(b), (d), (f), (h)] panels of Fig. 1, highlighted in red. This can also be easily inferred from Fig. 2. Here, for the different twist angles, we show the out-of-plane displacements of the atoms positioned onto or closest to the unit-cell long and short diagonals. These curves have been smoothened. However, particularly for large moiré cells, they show height profiles very close to those obtained along a zigzag cut (long diagonal) or armchair cut (short diagonal), except for small fluctuations due do the mismatch between the discrete lattice positions and the cell diagonals. Because the starting configuration is that of two twisted but flat graphene planes, these displacements are visualized, in each graphene plane, as a difference of the $z$ coordinate of each atom and the average $z$ in that plane (highlighted with a thin solid line). We can clearly distinguish how the two atoms (one for each plane) on the cell corners preserve their initial AA stacking, with their final distance estimated to be 


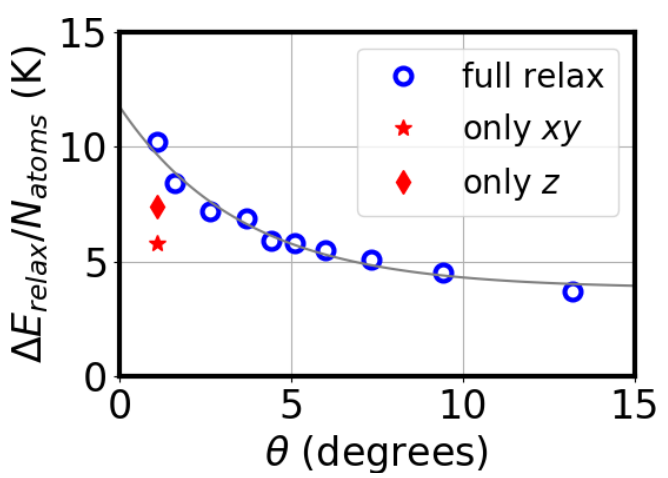

(a)

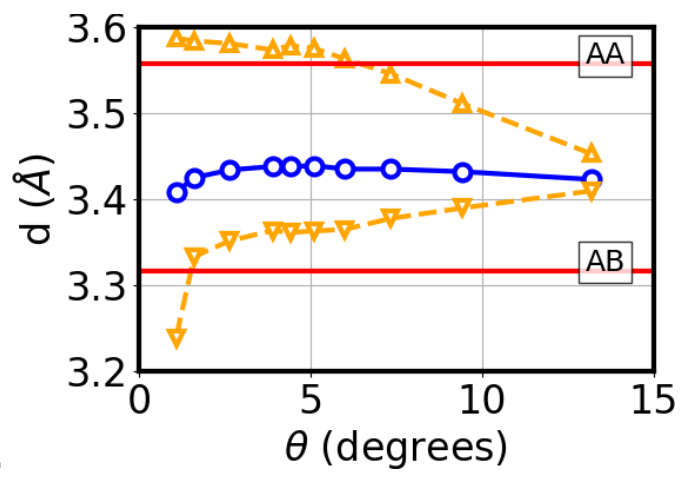

FIG. 4. (a) The per-atom relaxation energy as a function of the twist angle (blue circles). The red star (diamond) refers to $\theta=1.08^{\circ}$ and shows the relaxation energy when only the $x-y(z)$ coordinates are allowed to relax. (b) The interplane distance as a function of the twist angle calculated as the difference between the averages of the $z$ coordinates in each plane (solid blue line and circles). The same distance calculated for the untwisted bilayer in both the AA and $\mathrm{AB}$ stacking is also highlighted by the red lines. The orange dashed lines and triangles correspond to the smallest $\left(d_{\min }\right)$ and largest $\left(d_{\max }\right)$ interplane distance (see text).

$3.58 \AA$ A. Correspondingly, we can estimate the distance $d$ between the two graphene planes as the difference between the averages of the $z$ coordinates in each plane. This is reported in Table I and shown in Fig. 4, where we can observe a reduced distance at small twist angles. From the latter figure we can also see that the interplane distance lies between the calculate interplane distances for the untwisted bilayer at $\mathrm{AA}$ and $\mathrm{AB}$ stacking (highlighted by the red solid lines).

The patterns in Fig. 1 show that AA stacking regions, where there is an enhanced distance between an atom in the top plane and the corresponding (closest) atom in the bottom one, alternate with regions with $\mathrm{AB}$ stacking, the latter being predominant. One would expect a smooth change of the displacements when moving from $\mathrm{AA}$ to $\mathrm{AB}$ regions. This naive prediction is verified only for the larger angles. For the smaller twist angles such smooth behavior is replaced by an oscillating pattern, resulting in an atomic corrugation, as clearly visible from the relief maps in Figs. 1(a)-1(d).

The out-of-plane displacements are accompanied with significant in-plane relaxations. The $(x, y)$ displacement field is shown in Fig. 3. Here the color map and the vector lengths are proportional to the displacement with respect to the unrelaxed twisted bilayer. Since the patterns look quite similar at different twist angles, only the result for $1.08^{\circ}$ is shown, whereas the color bars distinguish the different systems. Interestingly enough, a vortexlike displacement field shows up in each plane, with the vorticity (intended as the curl of the displacement field) changing sign when moving from the top to the bottom plane and vice versa. Such a result can be explained by considering that $\mathrm{AB}$ stacking regions minimize the total energy of the system. Hence, close to an AA stacking configuration, the atoms of the two layers tend to move in their plane in opposite directions in order to minimize the overlap of their orbitals that is maximum at AA stacking. Indeed, it is observed that (i) the in-plane displacement is exactly null for the AA stacked pair of atoms (no arrow and blue region at the unit-cell corners in the figure); (ii) the maximum displacements are observed around the unit-cell corners, thus for the atoms that mostly feel the "repulsion" due to a stacking that is quite close to AA; and (iii) no displacement is observed in the $\mathrm{AB}$ stacking regions, as it can be evinced by the blue, hexagonal regions. While the displacement pattern looks quite similar for all the twist angles (at variance with the outof-plane displacements), the magnitude of the displacements decreases by an order of magnitude when moving from the smallest to the largest twist angle (the maximum displacement being of the order of $\sim 0.1 \AA$ and $\sim 0.01 \AA$ in the two cases, respectively).

The relaxation energy is defined as the energy gained by the structure when it is allowed to relax with respect to the unrelaxed configuration, in which the atoms are arranged in ideal honeycomb lattices in two parallel planes. It is reported in Table I. Such gain is extensive, i.e., it depends on the number of atoms in the unitary cells. In order to allow for a fair comparison between different twist angles, in Table I we also report the energy gain per atom. The relaxation energy normalized to the number of atoms in the unit cell is plotted in Fig. 4(a) as a function of the twist angle (blue dots). In addition to the four angles in Table I, in order to extend our dataset, we represent also points relative to the angles $4.41^{\circ}, 5.09^{\circ}, 6.01^{\circ}, 7.34^{\circ}, 9.43^{\circ}, 13.17^{\circ}$ [corresponding to $(n, m)=(8,7),(7,6),(6,5),(5,4),(4,3),(3,2)$, respectively].

The per-atom relaxation energy increases from 3.7 to $10.2 \mathrm{~K}$ when passing from the largest twist angle (corresponding to the smallest unit cell) to the smallest twist angle (corresponding to the largest unit cell). Such relaxation energy is an estimate of the upper bound of the thermal energy that in a real experiment would induce thermal fluctuations of the atomic positions that in turn would destroy the ground-state geometry pattern shown in Figs. 1 and 3. In the case of the $1.08^{\circ}$ magic angle, it has to be compared, for instance, with the critical temperature at which zero-resistance states are observed $\left(T_{c} \leqslant 1.7 \mathrm{~K}[2]\right)$ or at which the correlated insulator behavior at half filling is experimentally observed $(T<$ $1.0 \mathrm{~K}$ [1]). Interestingly, the relaxation energy increases upon decreasing the twist angle. This is explained by considering that in the case of large unit cells the atoms have more freedom to relax to lower total energy. In Fig. 4(b) we also show (solid blue line and circles) the interplane distance (averaged over the unit cell). It is almost halfway between the distance of the $\mathrm{AA}$ and the $\mathrm{AB}$ bilayer. However, as we have already noticed before, relaxation mechanisms tend to enlarge the "ef- 
fectively" $\mathrm{AB}$ regions; hence the average distance is slightly closer to that of the AB stacking. In the same figure, we report, as dashed orange lines and triangles, the smallest $d_{\min }$ and largest $d_{\max }$ interplane distances. The former (latter) is defined as the distance between the lowermost (uppermost) atom in the top plane and uppermost (lowermost) atom in the bottom plane. We can easily observe that they show a nontrivial dependence on the twist angle, especially in the low-twist-angle limit. This is, again, a consequence of the nontrivial relaxation pattern that shows up in this limit; such a pattern, while locally favoring the formation of $\mathrm{AB} / \mathrm{BA}$ stacked regions, when observed on the whole unit-cell surface corresponds to neither AA-stacked nor AB-stacked graphene planes. It should be pointed out that while such analysis was partially carried out by Uchida et al. [46], they only performed fully $a b$ initio calculations at twist angles $\theta>2^{\circ}$ and then extrapolated (using Fourier transform) the calculated geometries at the smaller angles. However, the present analysis shows that new oscillations (that would result in new Fourier components) in the relaxation pattern just show up at twist angles $\theta \simeq 2^{\circ}$ [see Fig. 2(b)] that might not be inferred from the calculations at the larger angles.

\section{A. Focus on the first magic angle at $\boldsymbol{\theta}=1.08^{\circ}$}

We now focus on TBG at the first magic angle, $\theta=1.08^{\circ}$, which shows the most intriguing and marked relaxation pattern. In the following we investigate the interplay between the out-of-plane and in-plane relaxations. This is done by performing two optimizations - the first by allowing all atoms to relax only along the $z$ direction and the second by allowing them to relax only their graphene planes (hence the $z$ coordinates are fixed while $x y$ coordinate are free to move). These partial relaxations have nontrivial effects on the electronic properties of the TBG.

First of all, we notice that these partial relaxations lead to a smaller gain in the total energy with respect to a full relaxation. This is shown by the red star and diamond in Fig. 4(a). This result is not surprising and is due to the reduced freedom of the atoms (constrained along $z$ or $x y$ ) to find optimal minimal energy configurations. Hence, neither the only- $x y$ nor the only- $z$ relaxations catch most of the relaxation energy (which for this system amount to $\sim 10 \mathrm{~K}$ ), which is due to an interplay between them. Such an interplay will be even more evident in the electronic properties, when we will discuss the energy gaps separating the FB manifold from the lower and higher energy bands.

\section{ELECTRONIC PROPERTIES}

In this section we discuss the electronic structure for the four angles analyzed, with and without including relaxation mechanisms. The results are summarized in Fig. 5, in which we plot the band structure at angles $\theta=3.698^{\circ}$ (panels a, $\mathrm{b}$ ), $\theta=2.65^{\circ}$ (panels c, d), $\theta=1.61^{\circ}$ (panels e, f), and $\theta=1.08^{\circ}$ (panels g-j). The (a), (c), (e), (g), (i) panels show the band structures including relaxation mechanisms, while in the (b), (d), (f), (h), (j) panels the atoms are fixed in their lattice positions. In Figs. 5(i) and 5(j) we show a zoom close to the Fermi energy in the case of the magic angle $\theta=1.08^{\circ}$.
The blue points correspond to the bands calculated using the DFT approach described in Sec. II. The red (full) lines are obtained within a tight-binding calculation where, however, the atomic positions for the relaxed structures are the ones optimized within the DFT approach. In general, we notice that there is an excellent agreement between the red (full) lines and the blue points. The DFT calculation could be well approximated by also adopting a continuum model (see Sec. II), where the interplane hoppings are parametrized, for the optimized structure, by coefficients $u=0.078 \pm 0.002$, $u^{\prime}=0.098 \pm 0.004$ and for unrelaxed ones by $u=u^{\prime}=$ $0.107 \pm 0.004$ almost independently of the angle. That can be adopted as a minimal single-particle description within more complex many-body $[11,50]$ approaches in order to study the superconductivity and the Mott insulating state of this system.

Inspection of Fig. 5 shows that by comparing the TB and the DFT curves, there is a tiny mismatch that can be evidenced, for instance, at $\Gamma$. This is due to the fact that, despite sharing the same atomic position, the two approaches do not account for electron-electron interaction in the same way. In particular, the tight-binding approach does not take into account any electron-electron interaction, while in the DFT approximation the electron-electron interaction is accounted for within the local density approximation. This discrepancy, at the $\Gamma$ point, could indeed be an estimate of the Hartree energy in TBG.

In all panels it is clearly visible that relaxation mechanisms tend to maximize the energy gaps at $\Gamma$ and, in particular, in the case of the magic angle $\theta=1.08^{\circ}$, the gap separating the FBs from the higher (lower) energy bands is about $26 \mathrm{meV}(16 \mathrm{meV})$, consistent with the experiments [1,2,8,1013]. On the other hand, those gaps cannot be reproduced at all if no relaxation is allowed. Aimed at identifying if there is a predominant role of the in-plane or of the out-of-plane displacements, these energy gaps have been estimated also when the system is allowed to relax only either in the $x-y$ plane (fixed $z$ coordinates) or along the $z$ axis (fixed $x-y$ coordinates). In the former case the two gaps turn out to both be zero; in the latter case we obtained gaps of $\sim 2$ and $\sim 14 \mathrm{meV}$, which underestimates these values in the fully relaxed system, especially in the hole side, but showing that a quite important role is played by the $z$ displacements. As far as the FB dispersion is concerned, we obtain a full bandwidth of $\sim 20 \mathrm{meV}$ in the relaxed system, which is of the order of that measured in the experiment of Ref. [2].

For the sake of completeness, in Fig. 6 we show the tight-binding band structure at $\theta=1.08^{\circ}$, calculated for the geometries as obtained from the fully ab initio calculation by allowing the atomic positions to relax only along the $z$ direction (a) or in the $x y$ plane (b). It is evident that, in agreement with the DFT outcome, while the in-plane relaxation does not substantially modify the band structure with respect to the unrelaxed system [compare Fig. 6(b) with Fig. 5(j)], the out-of-plane relaxation is mainly responsible for the band-gap opening. As such, the oscillating pattern previously discussed for the atomic displacements along the $z$ direction is a key factor to obtain a correct description of the low-energy dispersion at small angles. 

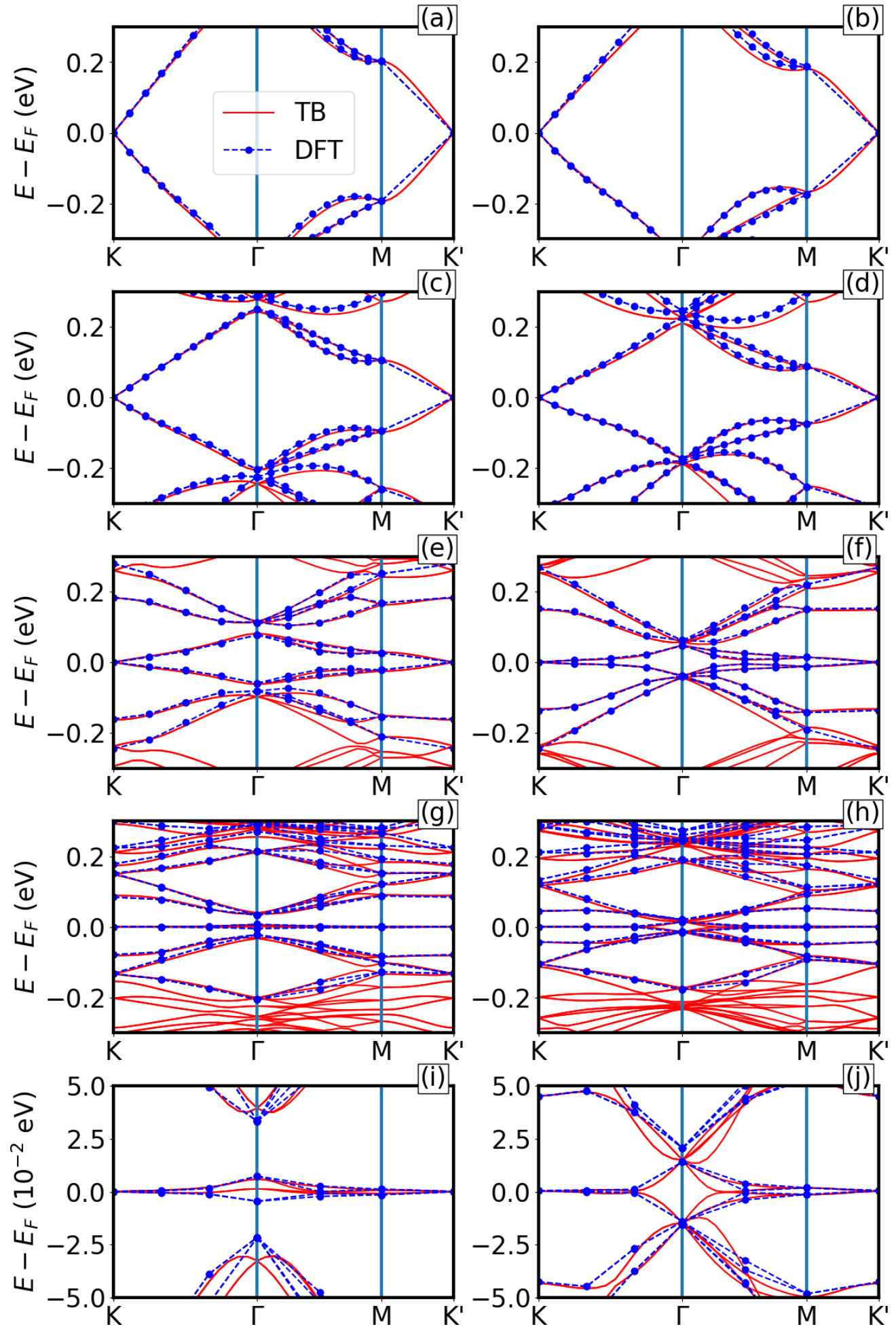

FIG. 5. Electronic band structure of TBG along the $K-\Gamma-M-K^{\prime}$ path at different twist angles: (a, b) $\theta=3.698^{\circ},(\mathrm{c}, \mathrm{d}) \theta=2.65^{\circ}$, (e, f) $\theta=1.61^{\circ},(\mathrm{g}, \mathrm{h}) \theta=1.08^{\circ}$, (i, j) $\theta=1.08^{\circ}$ zoomed around the Fermi energy (notice the different scale on the $y$ axis). The (a), (c), (e), (g), (i) panels correspond to the relaxed structure and the (b), (d), (f), (h), (j) ones to the initial, unrelaxed geometry. Blue dashed lines and filled dots correspond to the DFT calculation, red solid lines to the TB approach. Zero energy corresponds to the Fermi energy. The effective continuum model mentioned in Sec. II can be also used to approximate the DFT calculations. The parameters $u, u^{\prime}$ are independent of the angle and, within a reasonable error, can be approximated as $u=u^{\prime}=0.107 \pm 0.004$ in the unrelaxed case and $u=0.078 \pm 0.002$, $u^{\prime}=0.098 \pm 0.004$ for relaxed structures. 
(a)

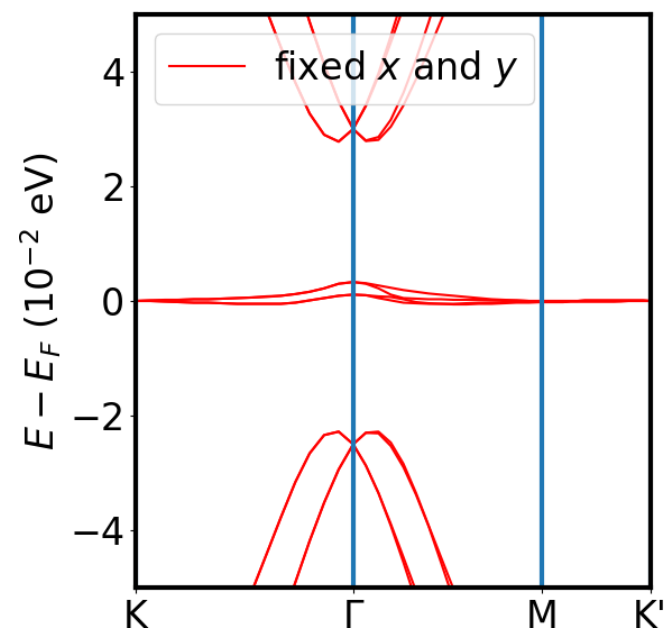

(b)

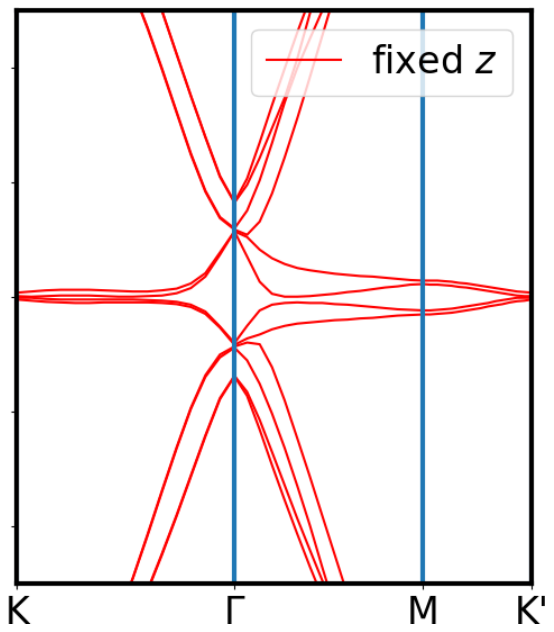

FIG. 6. Tight-binding electronic band structure of TBG along the $K-\Gamma-M-K^{\prime}$ path at $\theta=1.08^{\circ}$, zoomed around the Fermi energy. The band structures corresponding to the geometry as obtained by allowing only (a) out-of-plane and (b) in-plane relaxations in the DFT calculation are shown.

\section{CONCLUSIONS}

The FBs in TBG at the first magic angle can be intimately related with the atomic displacements arising as an effect of the interlayer vdW interaction. Large-scale first-principles calculations allow us to conclude that experimental gaps cannot be reproduced if we consider a flat bilayer system. Partial relaxation processes, with only in-plane or out-of-plane displacements, are also insufficient to obtain a proper description of the expected gaps and bandwidths. However, the main contribution to the band-gap opening at the $\Gamma$ point is due to the relaxations perpendicular to the graphene planes, i.e., at fixed $x-y$ coordinates. Out-of-plane relaxations are characterized by a strongly oscillating pattern that is smoothed (until it disappears) at large twist angles. On the other hand, the in-plane displacements show a vortexlike configuration, where the vorticity assumes opposite values in the two planes. However, the magnitude of these displacements decreases upon increasing the twist angle. Overall, the combination of the two patterns allows the atoms to override the steric repulsion felt by the $p_{z}$ orbitals, maximizing the regions with $\mathrm{AB}$ stacking at the expense of the regions showing AA stacking.

The larger the energy gain induced by the relaxation, the smaller the twist angle, decreasing by increasing the twist angle and eventually seeming to reach a plateau of $\sim 6.8 \mathrm{~K}$ at large twist angles. The smaller angles correspond to larger unit cells that can more easily accommodate the atomic rearrangement, and corresponding to large energy gain, of the order of $\sim 10 \mathrm{~K}$, much larger than the temperature at which the most exotic phenomena such as the correlated insulating phase and superconductivity are detected. Since the latter are intimately related to the presence of the FBs, that in turn we demonstrate being related with the atomic relaxation, such temperature $(10 \mathrm{~K})$ should be considered as an upper limit. Higher temperatures would destroy relaxation effects due to thermal atomic oscillations, and as a consequence, FB effects would be hindered.

By including relaxation effects we not only reproduce band gaps consistent with those measured in experiments, but we also give the effective parameters of a low-energy continuum model to be adopted for further investigation, including correlation effects. Interestingly enough, despite the fact that relaxation patterns have different shapes at various angles, the interplane hopping coefficients $u, u^{\prime}$ are found to be almost independent of the twist angle. That could be a useful hint to apply the continuum model also to smaller angles, where the unit cell would become impractically large to be addressed with atomistic approaches.

\section{ACKNOWLEDGMENTS}

We thank A. Stroppa and K. Yananose for stimulating discussions. We acknowledge use of the Monsoon2 system, a collaborative facility supplied under the Joint Weather and Climate Research Program, a strategic partnership between the UK Met Office and the Natural Environment Research Council.

G.C. and P.L. contributed equally to this work.
[1] Y. Cao, V. Fatemi, A. Demir, S. Fang, S. L. Tomarken, J. Y. Luo, J. D. Sanchez-Yamagishi, K. Watanabe, T. Taniguchi, E. Kaxiras, R. C. Ashoori, and P. Jarillo-Herrero, Nature (London) 556, 80 (2018).

[2] Y. Cao, V. Fatemi, S. Fang, K. Watanabe, T. Taniguchi, E. Kaxiras, and P. Jarillo-Herrero, Nature (London) 556, 43 (2018).
[3] E. Codecido, Q. Wang, R. Koester, S. Che, H. Tian, R. Lv, S. Tran, K. Watanabe, T. Taniguchi, F. Zhang, M. Bockrath, and C. N. Lau, Sci. Adv. 5, eaaw9770 (2019).

[4] X. Lu, P. Stepanov, W. Yang, M. Xie, M. A. Aamir, I. Das, C. Urgell, K. Watanabe, T. Taniguchi, G. Zhang, A. Bachtold, A. H. MacDonald, and D. K. Efetov, Nature (London) 574, 653 (2019). 
[5] A. L. Sharpe, E. J. Fox, A. W. Barnard, J. Finney, K. Watanabe, T. Taniguchi, M. A. Kastner, and D. GoldhaberGordon, Science 365, 605 (2019).

[6] M. Yankowitz, S. Chen, H. Polshyn, Y. Zhang, K. Watanabe, T. Taniguchi, D. Graf, A. F. Young, and C. R. Dean, Science 363, 1059 (2019).

[7] D. M. Kennes, L. Xian, M. Claassen, and A. Rubio, Nat. Commun. 11, 1124 (2020).

[8] Y. Choi, J. Kemmer, Y. Peng, A. Thomson, H. Arora, R. Polski, Y. Zhang, H. Ren, J. Alicea, G. Refael, F. von Oppen, K. Watanabe, T. Taniguchi, and S. Nadj-Perge, Nat. Phys. 15, 1174 (2019).

[9] R. Bistritzer and A. H. MacDonald, Proc. Natl. Acad. Sci. 108, 12233 (2011).

[10] A. Kerelsky, L. J. McGilly, D. M. Kennes, L. Xian, M. Yankowitz, S. Chen, K. Watanabe, T. Taniguchi, J. Hone, C. Dean, A. Rubio, and A. N. Pasupathy, Nature (London) 572, 95 (2019).

[11] Y. Xie, B. Lian, B. Jäck, X. Liu, C.-L. Chiu, K. Watanabe, T. Taniguchi, B. A. Bernevig, and A. Yazdani, Nature (London) 572, 101 (2019)

[12] Y. Jiang, X. Lai, K. Watanabe, T. Taniguchi, K. Haule, J. Mao, and E. Y. Andrei, Nature (London) 573, 91 (2019).

[13] S. Lisi, X. Lu, T. Benschop, T. A. de Jong, P. Stepanov, J. R. Duran, F. Margot, I. Cucchi, E. Cappelli, A. Hunter, A. Tamai, V. Kandyba, A. Giampietri, A. Barinov, J. Jobst, V. Stalman, M. Leeuwenhoek, K. Watanabe, T. Taniguchi, L. Rademaker et al., Nat. Phys. (2020), doi: 10.1038/s41567-020-01041-x.

[14] A. O. Sboychakov, A. V. Rozhkov, A. L. Rakhmanov, and F. Nori, Phys. Rev. B 100, 045111 (2019).

[15] L. Rademaker, D. A. Abanin, and P. Mellado, Phys. Rev. B 100, 205114 (2019).

[16] Y. W. Choi and H. J. Choi, Phys. Rev. B 98, 241412(R) (2018).

[17] M. Angeli, E. Tosatti, and M. Fabrizio, Phys. Rev. X 9, 041010 (2019).

[18] M. Koshino and Y.-W. Son, Phys. Rev. B 100, 075416 (2019).

[19] M. Lamparski, B. V. Troeye, and V. Meunier, 2D Mater. 7, 025050 (2020).

[20] E. F. Talantsev, R. C. Mataira, and W. P. Crump, Sci. Rep. 10, 212 (2020)

[21] I. Maity, M. H. Naik, P. K. Maiti, H. R. Krishnamurthy, and M. Jain, Phys. Rev. Research 2, 013335 (2020).

[22] F. Conte, D. Ninno, and G. Cantele, Phys. Rev. B 99, 155429 (2019).

[23] M. H. Naik and M. Jain, Phys. Rev. Lett. 121, 266401 (2018).

[24] F. Conte, D. Ninno, and G. Cantele, Phys. Rev. Research 2, 033001 (2020).

[25] R. Ribeiro-Palau, C. Zhang, K. Watanabe, T. Taniguchi, J. Hone, and C. R. Dean, Science 361, 690 (2018).

[26] A. K. Geim and I. V. Grigorieva, Nature (London) 499, 419 (2014).
[27] G. Cantele and D. Ninno, Phys. Rev. Materials 1, 014002 (2017).

[28] I. Borriello, G. Cantele, and D. Ninno, Nanoscale 5, 291 (2012).

[29] G. Cantele, Y.-S. Lee, D. Ninno, and N. Marzari, Nano Lett. 9 , 3425 (2009).

[30] P. Novelli, I. Torre, F. H. L. Koppens, F. Taddei, and M. Polini, Phys. Rev. B 102, 125403 (2020).

[31] V. N. Do, H. A. Le, and D. Bercioux, Phys. Rev. B 99, 165127 (2019).

[32] Z. Song, Z. Wang, W. Shi, G. Li, C. Fang, and B. A. Bernevig, Phys. Rev. Lett. 123, 036401 (2019).

[33] K. Hejazi, C. Liu, H. Shapourian, X. Chen, and L. Balents, Phys. Rev. B 99, 035111 (2019).

[34] J. Liu, J. Liu, and X. Dai, Phys. Rev. B 99, 155415 (2019).

[35] M. Z. Hasan and C. L. Kane, Rev. Mod. Phys. 82, 3045 (2010).

[36] D. Bercioux and P. Lucignano, Rep. Prog. Phys. 78, 106001 (2015).

[37] P. Lucignano, R. Raimondi, and A. Tagliacozzo, Phys. Rev. B 78, 035336 (2008)

[38] F. Guinea and N. R. Walet, Phys. Rev. B 99, 205134 (2019).

[39] M. Angeli, D. Mandelli, A. Valli, A. Amaricci, M. Capone, E. Tosatti, and M. Fabrizio, Phys. Rev. B 98, 235137 (2018).

[40] N. N. T. Nam and M. Koshino, Phys. Rev. B 96, 075311 (2017),

[41] F. Gargiulo and O. V. Yazyev, 2D Mater. 5, 015019 (2018).

[42] S. Dai, Y. Xiang, and D. J. Srolovitz, Nano Lett. 16, 5923 (2016).

[43] K. Zhang and E. B. Tadmor, J. Mech. Phys. Solids 112, 225 (2018).

[44] S. Carr, S. Fang, and E. Kaxiras, Nat. Rev. Mater. 5, 748 (2020).

[45] S. Carr, D. Massatt, S. B. Torrisi, P. Cazeaux, M. Luskin, and E. Kaxiras, Phys. Rev. B 98, 224102 (2018).

[46] K. Uchida, S. Furuya, J.-I. Iwata, and A. Oshiyama, Phys. Rev. B 90, 155451 (2014).

[47] M. Koshino and N. N. T. Nam, Phys. Rev. B 101, 195425 (2020).

[48] S. Carr, S. Fang, Z. Zhu, and E. Kaxiras, Phys. Rev. Research 1, 013001 (2019).

[49] P. Lucignano, D. Alfè, V. Cataudella, D. Ninno, and G. Cantele, Phys. Rev. B 99, 195419 (2019).

[50] M. Koshino, N. F. Q. Yuan, T. Koretsune, M. Ochi, K. Kuroki, and L. Fu, Phys. Rev. X 8, 031087 (2018).

[51] G. Kresse and J. Furthmüller, Phys. Rev. B 54, 11169 (1996).

[52] I. Hamada, Phys. Rev. B 89, 121103(R) (2014).

[53] P. E. Blöchl, Phys. Rev. B 50, 17953 (1994).

[54] G. Kresse and D. Joubert, Phys. Rev. B 59, 1758 (1999).

[55] S. Shallcross, S. Sharma, E. Kandelaki, and O. A. Pankratov, Phys. Rev. B 81, 165105 (2010).

[56] P. Moon and M. Koshino, Phys. Rev. B 87, 205404 (2013).

[57] J. M. B. Lopes dos Santos, N. M. R. Peres, and A. H. Castro Neto, Phys. Rev. Lett. 99, 256802 (2007). 